\title{
FUNCTIONAL ASSESSMENT ON THE PATIENTS WITH ADOLESCENT IDIOPATHIC SCOLIOSIS
}

\author{
Aisel Oseku, Evgeniya Dimitrova \\ National Sports Academy „Vassil Levski”, Sofia, Bulgaria
}

\begin{abstract}
Introduction: The aims of our study were to identify the available functional examination tests (FET) and patient reported outcome measures (PROM) instruments designed for spinal deformity to analyze and evaluate them and to develop a methodology for functional assessment of patients with adolescent idiopathic scoliosis (AIS).

Methods: A comprehensive search for all available FET and PROM and published review articles for spinal deformity was undertaken on PubMed, PEDRO, Google Scholar, Physiopedia up to December 2018. All full-text articles reporting evaluation, validation, surgical, orthotic, and/or physiotherapeutic scoliosis specific exercises (PSSE) treatment outcomes of these spinal deformities FET and PROM were retrieved and analyzed by us and methodology for functional assessment of patients with AIS was developed. The methodology includes the following methods and tests: inspection for trunk symmetry, Adams forward bend test, angle of trunk rotation (ATR) measured by scoliometer, muscle endurance tests, Spinal Appearance Questionnaire (SAQ) and Cobb angle measured on radiography.

Results and discussion: For a period of 1 year we accessed children with AIS. All children make Schroth PSSE. The analysis of the results confirms the positive effect of the PSSE to improve muscle endurance, balance and correction of the scoliosis. Within a month from the beginning, patients reported no back pain and within 2 months - a respiratory improvement, an enlargement of the chest, a reduced Cobb angle, an increased vitality. PSSE seem to have positive effects by reducing the symptoms and improving functions and body asymmetries.

Conclusion: A methodology for functional examination of children with AIS allows objective monitoring of the results of PSSE. Schroth method is effective of preventing the scoliosis' progression.
\end{abstract}

Key words: adolescent idiopathic scoliosis, functional assessment, physiotherapy.

\section{INTRODUCTION}

Scoliosis is defined as a lateral curvature of the spinal cord with Cob angle of $10^{\circ}$ or more. Scoliosis usually develops before puberty and leads to a 3 -dimensional deformation of the trunk. Compared to healthy individuals, patients with idiopathic scoliosis have reduced overall and asymmetric balance of the transversus abdominal muscles.

Schroth exercises are 3-dimensional exercises used for the treatment of scoliosis, (which were developed by Katharina Schroth in 1920); these provide sensors and kinesthetic stimulation, correcting abnormal breathing patterns present in patients with scoliosis. This program it also includes training to help patients consciously maintain correct behavior in activities of daily living.

Healthcare interventions are under increasing scrutiny regarding effectiveness. Functional examination tests (FET) and patient reported outcome measures (PROM) have revolutionized clinical research facilitating objective interpretation and comparison across different healthcare systems.
The objectives of our study were to identify the available functional examination tests and PROM instruments and questionnaires designed for spinal deformity to analyze and evaluate them and to develop a methodology for functional assessment of patients with adolescent idiopathic scoliosis.

In this study, stretching, Schroth and strengthening exercises were applied sequentially in children with idiopathic scoliosis to evaluate their effects on the Cobb angle and on the ribs.

\section{METHODS}

A comprehensive search for all available FET and PROM and published review articles for spinal deformity was undertaken on PubMed, PEDRO, Google Scholar, Physiopedia up to December 2018. All published full-text articles reporting evaluation, validation, surgical, orthotic, and/or physiotherapeutic scoliosis specific exercises treatment outcomes of these spinal deformities FET and PROM were retrieved and analyzed by us and methodology for functional assessment of patients with adolescent idiopathic scoliosis was developed. 
The level of evidence in conservative management of AIS is not too high. The treatments applied include surgery (instrumentation and fusion), stopping progression, or exercises. In the past, electrical stimulation has also been used, but without any significant results. Other treatments that are not recommended by current guidelines include manipulations and scars to change gait.

For a period of 1 year we accessed 45 children's with adolescent idiopathic scoliosis (AIS) with Cobb angle $>20^{\circ}$ and documented curve progression during growth (at least $4^{\circ} \mathrm{Cobb}$ ). We included ( $23 \mathrm{fe}$ males and 22 males) ranged in age between 15 and 18 years old with a mean age of $16.5 \pm 2.8$ years. They were distributed as follows: single thoracic 7
(15.3\%), single thoraco-lumbar 14 (30.8\%), single lumbar $10(23.1 \%)$ and double major (thoracic and lumbar) 14 (30.8\%).

All patients we taught to make exercises for correction according to Schroth method after the initial evaluation. All patients had to practice their exercises regularly for at least 4 months.

Patients were evaluated at least 6 months after their first evaluation. X-ray of the spine after 6 months of therapy at least.

To evaluate the effect of treatment, we considered the last $\mathrm{x}$-rays available for each single patient (Figure 1).

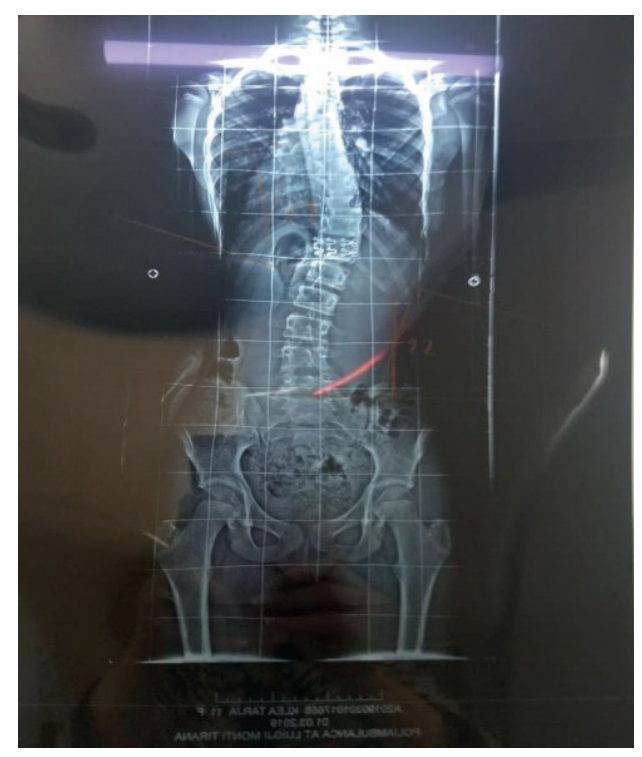

Figure 1. X-ray of patient.

Angle of trunk rotation (ATR) - rib hump (difference in elevation between right and left chest) was averaged over each individual $15^{\circ}$, as measured by Adam's flexion test using a scoliometer, during which the patient grasped her two hands together while in a standing position, naturally he slid his hands between his legs and leaned forward at $90^{\circ}$. We utilized the SAQ (Spinal Appearance Questionnaire) for every single patient.

The exercise program used in this study consisted of 3 phases and was implemented 3 times a week, for 12 weeks. The first stage was made up of stretches for 10 minutes to relax tight muscles and improve joint flexibility. The second phase consisted of Schroth exercises. Schroth Breathing (mostly rotational breathing), mostly the basic Schroth Exercise, was used to correct breathing patterns, The third phase was a soothing phase which consists of 10 minutes of muscle strengthening exercises to activate muscles that are corrected using Schroth exercises and for maintained the skeleton.

\section{RESULTS AND DISCUSSION}

The analysis of the research results after the treatment course confirms the positive effect of the physiotherapy program to improve muscle endurance, balance and correction of the scoliosis.

Within a month from the beginning, patients reported no back pain.

Within 2 months, they reported a respiratory improvement. Patients also benefited from an enlargement of the chest, a reduced scoliosis curve angle (measured in Cobb degrees), and an increased 
vitality. Corrective, therapeutic exercises seem to have positive effects by reducing the symptoms and improving function at different angles and body asymmetries.

After the 12-week intervention, the statistical processing of the data shows a significant Cobb angles and ATR improvement. The Cobb angle decreased from $18.51^{\circ} \pm 3.9^{\circ}$ to $14.35^{\circ} \pm 3.3^{\circ}, \Delta \mathrm{X}=4.16^{\circ}, \mathrm{p}<0.05$, and the ATR (rib hump) decreased from $15.3^{\circ} \pm 6.9$ to $9.1^{\circ} \pm 3.5, \Delta \mathrm{X}=6.2^{\circ}, \mathrm{p}<0.05$.

In this study, the successive application of the extension, Schroth methodology, and strengthening exercises lowered the patient's Cobb's angle and rib hump. To treat scoliosis, extend muscles should be shortened, and muscles shortened should be extended again to restore the shape of the spine. Stretching exercises implemented in the first stage may have relaxed the muscles that were asymmetrically shortened around the spine and stretched the muscles around the trunk, increasing flexibility. Zakaria et al. (2012) showed that stretching exercises reduced muscle spasms in the concave side and hyperactivity and corrected bending of the lumbar region extending shortened muscles.

\section{CONCLUSION}

According to current evidence, Schroth's methodology may be useful assistance to adolescent patients with idiopathic scoliosis. However, we need more evidence and are awaiting the outcome of consistently controlled trials with high expectations. The exercises proved to be superior to AIS, and should be considered a potential tool for the curve control. A methodology for functional examination of children with adolescent idiopathic scoliosis allows objective monitoring of the results of physiotherapeutic scoliosis specific exercises. Schroth method is an effective method of preventing the scoliosis' progression.

\section{REFERENCES}

Green, BN., Johnson, C., Moreau, W. (2009). Is physical activity contraindicated for individuals with scoliosis? A systematic literature review. J Chiropr Med, 8: 25-37. [Medline] [CrossRef]

Lehnert-Schroth, C. (2005). Three-dimensional Treatment for scoliosis. A physiotherapeutic method to improve deformities of the spine. Palo Alto: The Martindale Press, 2007.

Linek, P., Saulicz, E., Wolny, T., et al. (2015). Ultrasound evaluation of the symmetry of abdominal muscles in mild adolescent idiopathic scoliosis. J Phys Ther Sci, 27: 465-468. [Medline] [CrossRef].

Mooney, V., Gulick, J., Pozos, R. (2000). A preliminary report on the effect of measured strength training in adolescent idiopathic scoliosis. J Spinal Disord, 13: 102107. [Medline] [CrossRef]

Negrini, S., Aulisa, AG., Aulisa, L. et al. (2014). 2011 SOSORT guidelines: Orthopaedic and Rehabilitation treatment of idiopathic scoliosis during growth. Scoliosis; $7: 3$

Negrini, S., Aulisa, L., Ferraro, C. et al. (2005). Italian guidelines on rehabilitation treatment of adolescents with scoliosis or other spinal deformities. Eura.Medicophys. 41:183-201.

Park, JY., Park, GD., Lee, SG., et al. (2013). The effect of scoliosis angle on center of gravity sway. J Phys Ther Sci, 25: 1629-1631. [Medline] [CrossRef]

Rowe, DE., Bernstein, SM., Riddick, MF. et al. (1997). A meta-analysis of the efficacy of non-operative treatments for idiopathic scoliosis. J.Bone Joint Surg.Am.; 79:664-74. Scoliosis Research Society. Scoliosis Research Society Adolescent Idiopathic Scoliosis - Treatment. (2014). Last access 8-2 2014.http://www.srs.org/professionals/conditions_and_treat-ment/adolescent_idiopathic_scoliosis/ treatment.hm.

Zakaria, A., Hafez, AR., Buragadda, et al. (2012). Stretching versus mechanical traction of the spine in treatment of idiopathic scoliosis. J Phys Ther Sci, 2012, 24: 11271131. [CrossRef] 